\title{
Explosions on a Variety of Scales
}

\author{
Lars Bildsten \\ Kavli Institute for Theoretical Physics, University of California, \\ Santa Barbara, CA 93106, USA \\ email: Bildsten@kitp.ucsb.edu \\ Invited Talk
}

\begin{abstract}
Summary. The theoretical community is beginning to appreciate (and predict) the potential diversity of explosive outcomes from stellar evolution, while the supernovæ surveys are finding new kinds of supernovæ. This talk described two such new supernovæ. The first are ultraluminous core collapse supernovæ with radiated energies approaching $10^{51} \mathrm{ergs}$. The talk went on to present our recent work that explains these events with late-time energy deposition from rapidly rotating, highly magnetized neutron stars: magnetars. It concluded with our theoretical work on helium shell detonations on accreting white dwarfs that predict a new class of supernovæ called ".Ia's". The first such candidate may well have been found by the Palomar Transient Factory.
\end{abstract}

\section{Transients with Pan-STARRS-1}

\section{Stephen Smartt (and the PSI Science Consortium)}

Astrophysics Research Centre, School of Mathematics and Physics, Queen's University Belfast, Belfast, BT7 1NN, UK

email: s.smartt@qub.ac.uk

Invited Talk

Summary. The Pan-STARRS1 sky survey began operations in May 2010. Since then we have discovered more than 4000 optical transients in the Medium Deep Fields (11 fields of approximately 7 square degrees). Several hundred have been confirmed with optical spectroscopy. The talk discussed the challenges involved in automating reliable difference imaging, object detection and classification. The science part of the talk then focussed on the discovery of high-redshift, very luminous supernovæ which appear to arise in faint hosts. 